\title{
Multiple Nontrivial Solutions for a Nonlocal Problem with Sublinear Nonlinearity
}

\author{
Zhigao Shi $\mathbb{D}^{1}$ and Xiaotao Qian $\mathbb{D}^{2}$ \\ ${ }^{1}$ Teaching and Research Department of Mathematics and Physics, Fujian Jiangxia University, Fuzhou 350108, China \\ ${ }^{2}$ Department of Basic Teaching and Research, Yango University, Fuzhou 350015, China
}

Correspondence should be addressed to Xiaotao Qian; qianxiaotao1984@163.com

Received 21 November 2020; Accepted 13 May 2021; Published 17 June 2021

Academic Editor: Sergey Shmarev

Copyright ( 2021 Zhigao Shi and Xiaotao Qian. This is an open access article distributed under the Creative Commons Attribution License, which permits unrestricted use, distribution, and reproduction in any medium, provided the original work is properly cited.

In this paper, we study the following nonlocal problem $\left\{\begin{array}{ll}-\left(a-b \int_{\Omega}|\nabla u|^{2} d x\right) \Delta u=\lambda u+f(x)|u|^{p-2} u, & x \in \Omega, \\ u=0, & x \in \partial \Omega,\end{array}\right.$ where $a, b>0$ are constants, $1<p<2, \lambda>0, f \in L^{\infty}(\Omega)$ is a positive function, and $\Omega$ is a smooth bounded domain in $\mathbb{R}^{N}$ with $N \geq 3$. By variational methods, we obtain a pair of nontrivial solutions for the considered problem provided $|f|_{\infty}$ is small enough.

\section{Introduction and Main Results}

This paper is concerned with the existence and multiplicity of nontrivial solutions for the following nonlocal problem with Dirichlet boundary value conditions:

$$
\begin{cases}-\left(a-b \int_{\Omega}|\nabla u|^{2} d x\right) \Delta u=\lambda u+f(x)|u|^{p-2} u, & x \in \Omega, \\ u=0, & x \in \partial \Omega,\end{cases}
$$

where $a, b>0,1<p<2, \lambda>0, f \in L^{\infty}(\Omega)$ is a positive function, and $\Omega$ is a smooth bounded domain in $\mathbb{R}^{N}$ with $N \geq 3$.

In the past two decades, the following Kirchhoff type problems with Dirichlet boundary value conditions

$$
\begin{cases}-\left(a+b \int_{\Omega}|\nabla u|^{2} d x\right) \Delta u=f(x, u), & x \in \Omega, \\ u=0, & x \in \partial \Omega,\end{cases}
$$

have attracted great attention of many researchers. Such problems are often viewed as nonlocal because of the appear- ance of the term $a+b \int_{\Omega}|\nabla u|^{2} d x$, which implies that (2) is no longer a pointwise identity. It is worthwhile pointing out that the equation in (2) arises in various models of physical and biological systems. Indeed, problem (2) is related to the stationary analogue of the following equation:

$$
\rho \frac{\partial^{2} u}{\partial t^{2}}-\left(\frac{P_{0}}{h}+\frac{E}{2 L} \int_{0}^{L}\left|\frac{\partial u}{\partial x}\right| d x\right) \frac{\partial^{2} u}{\partial x^{2}}=0
$$

which was first presented by Kirchhoff [1] as an extension of the classical d'Alembert wave equation for free vibrations of elastic strings, where $u=u(x, t)$ denotes the lateral displacement, $\rho$ the mass density, $P_{0}$ the initial tension, $h$ the crosssection area, $E$ the Young modulus of the material, and $L$ the length of the string. Under different assumptions on $f(x, u)$, many interesting results on the existence of solutions to (2) were obtained. We refer the interested readers to [2-14] and the references therein.

However, we now face a new nonlocal term $a-b \int_{\Omega}$ $|\nabla u|^{2} d x$, which is different from the well-known Kirchhoff type nonlocal term $a+b \int_{\Omega}|\nabla u|^{2} d x$. Now, there has been some results on the existence and multiplicity of nontrivial solutions to this new nonlocal problem (see [15-22]). 
In particular, Yin and Liu [15] firstly studied this kind of problem:

$$
\begin{cases}-\left(a-b \int_{\Omega}|\nabla u|^{2} d x\right) \Delta u=|u|^{p-2} u, & x \in \Omega, \\ u=0, & x \in \partial \Omega,\end{cases}
$$

where $2<p<2^{*}$, and obtained the existence and multiplicity of solutions for the problem.

In [16], Lei et al. considered

$$
\begin{cases}-\left(a-b \int_{\Omega}|\nabla u|^{2} d x\right) \Delta u=f_{\lambda}(x)|u|^{p-2} u, & x \in \Omega, \\ u=0, & x \in \partial \Omega,\end{cases}
$$

where $1<p<2$, and proved under certain condition on $f_{\lambda}(x)$ , that there are at least two positive solutions. After this, the authors also studied the problem with singularity [17].

Wang et al. [20] investigated the nonlocal problem with critical exponent

$$
\left\{\begin{array}{l}
-\left(a-b \int_{\mathbb{R}^{4}}|\nabla u|^{2} d x\right) \Delta u=|u|^{2} u+\mu g(x), \quad x \in \mathbb{R}^{4}, \\
u \in \mathscr{D}^{1,2}\left(\mathbb{R}^{4}\right) .
\end{array}\right.
$$

When $\mu$ is a nonnegative parameter and $g(x) \in L^{4 / 3}\left(\mathbb{R}^{4}\right)$, they showed the existence of multiple positive solutions.

Recently, Zhang and Zhang [22] studied the nonlocal problem

$$
\begin{cases}-\left(a-b \int_{\Omega}|\nabla u|^{2} d x\right) \Delta u=\lambda u+g(x, u), & x \in \Omega, \\ u=0, & x \in \partial \Omega,\end{cases}
$$

where the parameter $0<\lambda<a \lambda_{1}, \lambda_{1}$ is the first eigenvalue of operator $-\Delta$, and $g$ is a superlinear function with subcritical growth. By using the Mountain Pass Theorem, the authors obtained the existence of a nontrivial solution.

As far as we know, there is no work on the existence of solution to (1), which is just our purpose here. Moreover, we extend $\lambda \geq a \lambda_{1}$ and without assuming nonlinearity is superlinear.

Our main result can be stated as follows.

Theorem 1. Assume that $a, b>0,1<p<2, \lambda>0, f \in L^{\infty}(\Omega)$ is a positive function, then problem (1) has at least a pair of nontrivial solutions if $|f|_{\infty}$ is small enough.

The paper is organized as follows. In Section 2, we give some notations and preliminaries. In Section 3, we prove Theorem 1 for the case of $0<\lambda<a \lambda_{1}$. Section 4 is devoted to the proof of Theorem 1 for the case of $\lambda \geq a \lambda_{1}$.

\section{Notations and Preliminaries}

Throughout this paper, we make use of the following notations. $H_{0}^{1}(\Omega)$ and $L^{q}(\Omega)$ are standard Sobolev spaces with the usual norm $\|u\|^{2}=\int_{\Omega}|\nabla u|^{2} d x,|u|_{q}^{q}=\int_{\Omega}|u|^{q} d x$. $B_{r}(x)\left(\partial B_{r}(x)\right)$ denotes an open ball (the sphere) centered at $x$ with radius $r>0 . \longrightarrow$ and $\rightarrow$ denote strong and weak convergence, respectively. For each $1 \leq r \leq 2^{*}$, we denote by $S_{q}$ the best Sobolev constant for the embedding of $H_{0}^{1}(\Omega)$ into $L^{q}(\Omega)$. Let $0<\lambda_{1}<\lambda_{2} \leq \cdots \leq \lambda_{k}<\cdots$ be the sequence of eigenvalues of $-\Delta$ on $H_{0}^{1}(\Omega)$ satisfying $\lim _{k \rightarrow \infty} \lambda_{k}=+\infty$, and let $e_{1}, e_{2}, \cdots$, be the corresponding orthonormal eigenfunctions in $L^{2}(\Omega)$.

By the Sobolev Theorem and $f \in L^{\infty}(\Omega)$, the functional

$$
I(u)=a 2\|u\|^{2}-b 4\|u\|^{4}-\lambda 2 \int_{\Omega}|u|^{2} d x-1 p \int_{\Omega} f(x)|u|^{p} d x
$$

is well defined on $H_{0}^{1}(\Omega)$. Furthermore, it belongs to $C^{1}\left(H_{0}^{1}\right.$ $(\Omega), \mathbb{R})$, and its critical points are precisely the weak solutions of (1). Here, we say $u \in H_{0}^{1}(\Omega)$ is a weak solution to (1), if for any $v \in H_{0}^{1}(\Omega)$, it holds

$$
\begin{aligned}
& \left(a-b \int_{\Omega}|\nabla u|^{2} d x\right) \int_{\Omega} \nabla u \nabla v d x-\lambda \int_{\Omega} u v d x-\int_{\Omega} f(x) \\
& \cdot|u|^{p^{-2}} u v d x=0 .
\end{aligned}
$$

Following [15], we first prove that the functional $I$ satisfies the $(P S)_{c}$ condition for any $c<a^{2} / 4 b$.

Lemma 2. Under the assumptions of Theorem 1, I satisfies the $(P S)_{c}$ condition with $c<a^{2} / 4 b$.

Proof. Let $\left\{u_{n}\right\} \subset H_{0}^{1}(\Omega)$ be a $(P S)_{c}$ sequence for $I$ with $c<$ $a^{2} / 4 b$; that is,

$$
I\left(u_{n}\right) \longrightarrow c, I^{\prime}\left(u_{n}\right) \longrightarrow 0 \text {, as } n \longrightarrow \infty
$$

By the Sobolev Theorem and (10),

$$
\begin{aligned}
c+1+o(1)\left\|u_{n}\right\| & \geq I\left(u_{n}\right)-\frac{1}{2}\left\langle I^{\prime}\left(u_{n}\right), u_{n}\right\rangle \\
& =\frac{b}{4}\left\|u_{n}\right\|^{4}-\left(\frac{1}{p}-\frac{1}{2}\right) \int_{\Omega} f(x)\left|u_{n}\right|^{p} d x \\
& \geq \frac{b}{4}\left\|u_{n}\right\|^{4}-\left(\frac{1}{p}-\frac{1}{2}\right)|f|_{\infty} S_{p}^{-p / 2}\left\|u_{n}\right\|^{p} .
\end{aligned}
$$

Since $p<2$, we conclude that $\left\{u_{n}\right\}$ is bounded in $H_{0}^{1}(\Omega)$. Up to a subsequence (still denoted by $\left\{u_{n}\right\}$ ), we may assume that 


$$
\left(\begin{array}{ll}
u_{n} \rightarrow u, & \text { in } H_{0}^{1}(\Omega), \\
u_{n} \longrightarrow u, & \text { in } L^{r}(\Omega), 1 \leq r<2^{*} \\
u_{n} \longrightarrow u, & \text { a.e.in } \Omega .
\end{array}\right.
$$

By using Hölder's inequality, it follows from (12) that

$$
\left.\left.\left|\int_{\Omega} f(x)\right| u_{n}\right|^{p-2} u_{n}\left(u_{n}-u\right) d x|\leq| f\right|_{\infty}\left|u_{n}\right|_{p}^{p-1}\left|u_{n}-u\right|_{p} \longrightarrow 0
$$

as $n \longrightarrow \infty$. Similarly, we also have

$$
\left|\int_{\Omega} u_{n}\left(u_{n}-u\right) d x\right| \leq\left|u_{n}\right|_{2}\left|u_{n}-u\right|_{2} \longrightarrow 0
$$

From the two above convergences, we get

$$
o(1)=I^{\prime}\left(u_{n}\right)\left(u_{n}-u\right)=\left(a-b\left\|u_{n}\right\|^{2}\right) \int_{\Omega} \nabla u_{n} \nabla\left(u_{n}-u\right)+o(1),
$$

as $n \longrightarrow \infty$. We claim that $a-b\left\|u_{n}\right\|^{2} \longrightarrow 0$ is false. If, to the contrary, namely, $\left\|u_{n}\right\|^{2} \longrightarrow a b$, define a functional by

$$
\phi(u)=\lambda 2 \int_{\Omega} u^{2} d x+1 p \int_{\Omega} f(x)|u|^{p} d x, \quad u \in H_{0}^{1}(\Omega) .
$$

Then,

$$
\left\langle\phi^{\prime}(u), v\right\rangle=\lambda \int_{\Omega} u v d x+\int_{\Omega} f(x)|u|^{p-2} u v d x, \quad u, v \in H_{0}^{1}(\Omega) .
$$

By using Hölder's inequality again, we obtain

$$
\begin{aligned}
& \left|\int_{\Omega} f(x)\left(\left|u_{n}\right|^{p-2} u_{n}-|u|^{p-2} u\right) v d x\right| \\
& \quad \leq\left.|f|_{\infty} \int_{\Omega}|| u_{n}\right|^{p-2} u_{n}-|u|^{p-2} u|| v \mid d x \\
& \quad \leq\left.|f|_{\infty}|| u_{n}\right|^{p-2} u_{n}-\left.|u|^{p-2} u\right|_{p / p-1} S_{p}^{-p / 2}\|v\|^{p}, \\
& \left|\int_{\Omega} u_{n} v-u v d x\right| \leq \int_{\Omega}\left|u_{n}-u\right||v| d x \leq\left|u_{n}-u\right|_{2} \lambda_{1}^{-1}\|v\|^{2} .
\end{aligned}
$$

Hence, by using (12), we obtain

$$
\begin{array}{r}
\left\|\phi^{\prime}\left(u_{n}\right)-\phi^{\prime}(u)\right\| \leq\left|u_{n}-u\right|_{2} \lambda_{1}^{-1}+\left.|f|_{\infty}|| u_{n}\right|^{p-2} u_{n} \\
-\left.|u|^{p-2} u\right|_{p / p-1} S_{p}^{-p / 2} \longrightarrow 0
\end{array}
$$

as $n \longrightarrow \infty$. This shows $\phi^{\prime}\left(u_{n}\right) \longrightarrow \phi^{\prime}(u)$.
On the other hand, from

$$
o(1)=\left\langle I^{\prime}\left(u_{n}\right), v\right\rangle=\left(a-b\left\|u_{n}\right\|^{2}\right) \int_{\Omega} \nabla u \nabla v d x-\left\langle\phi^{\prime}\left(u_{n}\right), v\right\rangle
$$

and $\left\|u_{n}\right\|^{2} \longrightarrow a / b$, we have $\phi^{\prime}\left(u_{n}\right) \longrightarrow 0$.

Thus, we can deduce that

$$
\left\langle\phi^{\prime}(u), v\right\rangle=\lambda \int_{\Omega} u v d x+\int_{\Omega} f(x)|u|^{p-2} u v d x=0, \quad \forall v \in H_{0}^{1}(\Omega) .
$$

By the variational method fundamental lemma [23], we further obtain

$$
\lambda u(x)+f(x)|u(x)|^{p-2} u(x)=0, \quad \text { a.e. } x \in \Omega .
$$

Since $f(x)>0$, it then follows that $u=0$.

By (12) and $f \in L^{\infty}(\Omega)$, we can use the Vitali Convergence Theorem to obtain

$$
\lim _{n \rightarrow \infty} \int_{\Omega} f(x)\left|u_{n}\right|^{p} d x=\int_{\Omega} f(x)|u|^{p} d x
$$

and consequently,

$$
\begin{aligned}
\phi\left(u_{n}\right) & =\frac{\lambda}{2} \int_{\Omega} u_{n}^{2} d x+\frac{1}{p} \int_{\Omega} f(x)\left|u_{n}\right|^{p} d x \\
& \longrightarrow \frac{\lambda}{2} \int_{\Omega}|u|^{2} d x+\frac{1}{p} \int_{\Omega} f(x)|u|^{p} d x=0 .
\end{aligned}
$$

This and $\left\|u_{n}\right\|^{2} \longrightarrow a / b$ provide

$$
\begin{aligned}
I\left(u_{n}\right)= & \frac{a}{2}\left\|u_{n}\right\|^{2}-\frac{b}{4}\left\|u_{n}\right\|^{4}-\frac{\lambda}{2} \int_{\Omega}\left|u_{n}\right|^{2} d x \\
& -\frac{1}{p} \int_{\Omega} f(x)\left|u_{n}\right|^{p} d x \longrightarrow \frac{a^{2}}{4 b},
\end{aligned}
$$

which contradicts $I\left(u_{n}\right) \longrightarrow c<a^{2} / 4 b$. Thus, the claim follows. In turn, we have from (15) that $\int_{\Omega} \nabla u_{n} \nabla\left(u_{n}-u\right) d x$ $\longrightarrow 0$, and hence, $\left\|u_{n}\right\| \longrightarrow\|u\|$. Combining this with the weak convergence of $\left\{u_{n}\right\}$ in $H_{0}^{1}(\Omega)$, we deduce that $u_{n}$ $\longrightarrow u$ in $H_{0}^{1}(\Omega)$.

\section{Proof of Theorem 1 for $0<\lambda<a \lambda_{1}$}

In this section, we will use the Mountain Pass Theorem to prove the existence of a pair of nontrivial solutions of the considered problem for $0<\lambda<a \lambda_{1}$.

Lemma 3. Assume that $0<\lambda<a \lambda_{1}$. If $|f|_{\infty}$ is sufficiently small, then there is a sequence $\left\{u_{n}\right\} \subset H_{0}^{1}(\Omega)$ such that $u_{n} \geq$ $0, I\left(u_{n}\right) \longrightarrow c_{*}$ and $I^{\prime}\left(u_{n}\right) \longrightarrow 0$, where $0<c_{*}<a^{2} / 4 b$. 
Proof. By the Sobolev Theorem, we have that

$$
\begin{aligned}
I(u) & =\frac{a}{2}\|u\|^{2}-\frac{b}{4}\|u\|^{4}-\frac{\lambda}{2} \int_{\Omega}|u|^{2} d x-\frac{1}{p} \int_{\Omega} f(x)|u|^{p} d x \\
& \geq \frac{1}{2}\left(a-\frac{\lambda}{\lambda_{1}}\right)\|u\|^{2}-\frac{b}{4}\|u\|^{4}-\frac{1}{p}|f|_{\infty} S_{p}^{-p / 2}\|u\|^{p} \\
& =\|u\|^{p}\left\{\frac{1}{2}\left(a-\frac{\lambda}{\lambda_{1}}\right)\|u\|^{2-p}-\frac{b}{4}\|u\|^{4-p}-\frac{1}{p}|f|_{\infty} S_{p}^{-p / 2}\right\} .
\end{aligned}
$$

For $A=1 / 2\left(a-\lambda / \lambda_{1}\right)$ and $B=b / 4$, the function $g:(0$, $+\infty) \longrightarrow \mathbb{R}$ defined by

$$
g(t):=A t^{2-p}-B t^{4-p}
$$

attains its maximum value at

$$
\rho=\left[\frac{(2-p) A}{(4-p) B}\right]^{1 / 2}
$$

Take $D_{0}=g(\rho)$ and note that, for any $u \in H_{0}^{1}(\Omega),\|u\|$ $=\rho$, there holds

$$
I(u) \geq \rho^{p}\left\{D_{0}-\frac{1}{p}|f|_{\infty} S_{p}^{-p / 2}\right\} \geq \frac{\rho^{p}}{2} \frac{D_{0}}{2}=: \theta>0,
$$

whenever

$$
|f|_{\infty} \leq \frac{D_{0} p}{2} S_{p}^{p / 2}
$$
that

On the other hand, let $u \in H_{0}^{1}(\Omega) \backslash\{0\}$, then we have

$$
\begin{aligned}
\lim _{t \longrightarrow+\infty} I(t u)= & \lim _{t \rightarrow+\infty}\left[\frac{a}{2} t^{2}\|u\|^{2}-\frac{b}{4} t^{4}\|u\|^{4}-\frac{\lambda}{2} t^{2} \int_{\Omega}|u|^{2} d x\right. \\
& \left.-\frac{1}{p} t^{p} \int_{\Omega} f(x)|u|^{p} d x\right]=-\infty .
\end{aligned}
$$

Thus, there exists $v_{1} \in H_{0}^{1}(\Omega)$ such that $\left\|v_{1}\right\|>\rho$ and $I$ $\left(v_{1}\right)<0$

By $I(u)=I(|u|)$, we may assume that $u_{n} \geq 0$. By applying the Mountain Pass Theorem without (PS) condition [24], we construct a sequence $\left\{u_{n}\right\} \subset H_{0}^{1}(\Omega)$ satisfying $I\left(u_{n}\right) \longrightarrow c_{*}$ and $I^{\prime}\left(u_{n}\right) \longrightarrow 0$ for

$$
c_{*}:=\inf _{\gamma \in \Gamma} \max _{t \in[0,1]} I(\gamma(t)) \geq \theta>0,
$$

where

$$
\Gamma:=\left\{\gamma \in C\left([0,1], H_{0}^{1}(\Omega)\right): \gamma(0)=0, \gamma(1)=v_{1}\right\}
$$

From easy calculations, we get

$$
\begin{aligned}
\max _{t \geq 0} I\left(t v_{1}\right)= & \max _{t \geq 0}\left\{\frac{a}{2} t^{2}\left\|v_{1}\right\|^{2}-\frac{b}{4} t^{4}\left\|v_{1}\right\|^{4}-\frac{\lambda}{2} t^{2} \int_{\Omega}\left|v_{1}\right|^{2} d x\right. \\
& \left.-\frac{1}{p} t^{p} \int_{\Omega} f(x)\left|v_{1}\right|^{p} d x\right\} \\
< & \max _{t \geq 0}\left\{\frac{a}{2} t^{2}\left\|v_{1}\right\|^{2}-\frac{b}{4} t^{4}\left\|v_{1}\right\|^{4}\right\} \leq \frac{a^{2}}{4 b} .
\end{aligned}
$$

This and the definition of $c_{*}$ yield that $0<c_{*}<a^{2} / 4 b$. Thus, we complete the proof of Lemma 3.

Proposition 4. Assume $a, b>0,0<\lambda<a \lambda_{1}$ and $f(x) \in L^{\infty}$ $(\Omega)$ is a positive function. Then, problem (1) admits a pair of nontrivial solutions if $|f|_{\infty}$ is small enough.

Proof. By Lemma 3, we obtain a sequence $\left\{u_{n}\right\} \subset H_{0}^{1}(\Omega)$ such that $u_{n} \geq 0, I\left(u_{n}\right) \longrightarrow c_{*}$ and $I^{\prime}\left(u_{n}\right) \longrightarrow 0$, provided $|f|_{\infty}$ is small enough. It then follows from Lemma 2 that there exists $u \in H_{0}^{1}(\Omega)$ such that $u_{n} \longrightarrow u$ in $H_{0}^{1}(\Omega)$ with $I(u)=c_{*}$ and $I^{\prime}(u)=0$, which implies that $u$ is a nontrivial nonnegative solution of (1). By the symmetry of functional $I$, we further deduce that $-u$ is a nontrivial nonpositive solution of (1). This completes the proof.

\section{Proof of Theorem 1 for $\lambda \geq a \lambda_{1}$}

Since $\lambda \geq a \lambda_{1}$, the method used in the previous section does not work here. Indeed, we shall apply the following Linking Theorem [25] to establish the existence of a pair of nontrivial solutions for problem (1) when $\lambda \geq a \lambda_{1}$.

Theorem 5. Let $X$ be a real Banach space with $X=Y \oplus Z$ and $\operatorname{dim} Y<+\infty$. Suppose that $I \in C^{l}(X, \mathbb{R})$ satisfies the following:

$\left(I_{1}\right)$ There are $\tilde{\rho}, \widetilde{\theta}>0$ such that $\left.I\right|_{\partial B_{\tilde{\rho}}(0) \cap Z} \geq \widetilde{\theta}$

$\left(I_{2}\right)$ There are $e \in \partial B_{\tilde{\rho}}(0) \cap Z$ and $R>\tilde{\rho}$ such that $\left.I\right|_{\partial M} \leq 0$ with $M=\left(B_{R}(0) \cap Y\right) \oplus\{t e: 0<t<R\}$

Then, there exists a sequence $\left\{u_{n}\right\} \subset X$ satisfying $I\left(u_{n}\right)$ $\longrightarrow c$ and $I^{\prime}\left(u_{n}\right) \longrightarrow 0$ for

$$
\tilde{c}_{*}:=\inf _{\tilde{\gamma} \in \Gamma} \max _{u \in M} I(\tilde{\gamma}(u))
$$

where

$$
\widetilde{\Gamma}:=\left\{\tilde{\gamma} \in C(M, X):\left.\tilde{\gamma}\right|_{\partial M}=i d\right\}
$$

As the sequence of eigenvalues $\lambda_{k}$ goes to infinity, there is $n \in \mathbb{N}$ such that $a \lambda_{1} \leq \lambda<a \lambda_{n+1}$. Set

$$
Y=\operatorname{span}\left\{e_{1}, \cdots, e_{n}\right\}, \quad Z=Y^{\perp} .
$$

Clearly, $X=Y \oplus Z$. 
Lemma 6. There exists $z \in Z \backslash\{0\}$ such that

$$
\max _{u \in Y \oplus \mathbb{R} z} I(u)<\frac{a^{2}}{4 b} .
$$

Proof. Since, for any $u \in H_{0}^{1}(\Omega) \backslash\{0\}$,

$$
\begin{aligned}
\max _{t \geq 0} I(t u)= & \max _{t \geq 0}\left\{\frac{a}{2} t^{2}\|u\|^{2}-\frac{b}{4} t^{4}\|u\|^{4}-\frac{\lambda}{2} t^{2} \int_{\Omega}|u|^{2} d x\right. \\
& \left.-\frac{1}{p} t^{p} \int_{\Omega} f(x)|u|^{p} d x\right\} \\
< & \max _{t \geq 0}\left\{\frac{a}{2} t^{2}\|u\|^{2}-\frac{b}{4} t^{4}\|u\|^{4}\right\} \leq \frac{a^{2}}{4 b},
\end{aligned}
$$

then there exists $u_{*} \in H_{0}^{1}(\Omega)$ such that

$$
z_{*} \in Z \backslash\{0\}, \max _{u \in Y \oplus \mathbb{R} z_{*}} I(u)<\frac{a^{2}}{4 b},
$$

where

$$
z_{*}=u_{*}-\sum_{k=1}^{n}\left(\int_{\Omega} u_{*} e_{k} d x\right) e_{k} .
$$

This completes the proof of Lemma 6.

Proposition 7. Assume $a, b>0, \lambda \geq a \lambda_{1}$ and $f(x) \in L^{\infty}(\Omega)$ is a positive function. Then, problem (1) admits a pair of nontrivial solutions if $|f|_{\infty}$ is small enough.

Proof. Firstly, we have for any $u \in Z$,

$$
\begin{aligned}
I(u) & =\frac{a}{2}\|u\|^{2}-\frac{b}{4}\|u\|^{4}-\frac{\lambda}{2} \int_{\Omega}|u|^{2} d x-\frac{1}{p} \int_{\Omega} f(x)|u|^{p} d x \\
& \geq \frac{1}{2}\left(a-\frac{\lambda}{\lambda_{n+1}}\right)\|u\|^{2}-\frac{b}{4}\|u\|^{4}-|f|_{\infty} \frac{1}{p} S_{p}^{-p / 2}\|u\|^{p},
\end{aligned}
$$

and therefore, as in the proof of Lemma 3, we can prove that $I$ satisfies the condition $\left(I_{1}\right)$ of Theorem 5 when $|f|_{\infty}$ is small.

Secondly, since $\lambda \geq a \lambda_{n}$, we also have for any $u \in Y$,

$$
\begin{aligned}
I(u) & =\frac{a}{2}\|u\|^{2}-\frac{b}{4}\|u\|^{4}-\frac{\lambda}{2} \int_{\Omega}|u|^{2} d x-\frac{1}{p} \int_{\Omega} f(x)|u|^{p} d x \\
& \leq \frac{1}{2}\left(a \lambda_{n}-\lambda\right)|u|_{2}^{2} \leq 0 .
\end{aligned}
$$

Moreover, if $z \in Z \backslash\{0\}$ is given by Lemma 6, we can apply the equivalence of norms in the finite dimensional space, to obtain for $u \in Y \oplus \mathbb{R} z$,

$$
I(u) \longrightarrow-\infty, \quad \text { as }\|u\| \longrightarrow+\infty .
$$

Thus, the condition $\left(I_{2}\right)$ is satisfied for $R$ large enough.
Finally, by Theorem 5, Lemma 6 , and $I(|u|)=I(u)$, we conclude that there is a sequence $\left\{u_{n}\right\} \subset H_{0}^{1}(\Omega)$ satisfying $u_{n} \geq 0, I\left(u_{n}\right) \longrightarrow \tilde{c}_{*}$ and $I^{\prime}\left(u_{n}\right) \longrightarrow 0$, provided $|f|_{\infty}$ is small enough. Then, we can argue as in the proof of Proposition 4 to obtain a pair of nontrivial solutions of (1). Thus, the proof of Proposition 7 is complete.

\section{Conclusions}

In this paper, we consider a new nonlocal problem different from the well-known Kirchhoff type problem. Compared with the existing literature, we extend $\lambda \geq a \lambda_{1}$ and without assuming nonlinearity is superlinear, which causes the methods employed in the previous papers cannot be directly applied here. To overcome this difficulty, we distinguish two cases: (1) $0<\lambda<a \lambda_{1}$ and (2) $\lambda \geq a \lambda_{1}$. In the former case, the existence of a nontrivial nonnegative solution is established via the Mountain Pass Theorem, while the Linking Theorem for the latter case. Furthermore, the symmetry of the corresponding functional brings us another nontrivial nonpositive solution.

\section{Data Availability}

No data were used to support this study.

\section{Conflicts of Interest}

The authors declare that they have no competing interests.

\section{Authors' Contributions}

All authors contributed equally and significantly in this paper. All authors read and approved the final manuscript.

\section{Acknowledgments}

This research was supported by the National Natural Science Foundation of China (11871152).

\section{References}

[1] G. Kirchhoff, Mechanik, Teubner, Leipzig, 1883.

[2] C. O. Alves, F. J. S. A. Corrêa, and T. F. Ma, "Positive solutions for a quasilinear elliptic equation of Kirchhoff type," Computers \& Mathematcs with Applications, vol. 49, no. 1, pp. 85-93, 2005.

[3] G. M. Figueiredo and G. G. dos Santos, "Existence of positive solution for Kirchhoff type problem with critical discontinuous nonlinearity," Annales Academiae Scientiarum Fennicae Mathematica, vol. 44, no. 2, pp. 987-1002, 2019.

[4] A. Fiscella and E. Valdinoci, "A critical Kirchhoff type problem involving a nonlocal operator," Nonlinear Analysis, vol. 94, pp. 156-170, 2014.

[5] W. Gan and S. Liu, "Multiple positive solutions of a class of Schrödinger-Poisson equation involving indefinite nonlinearity in $\mathbb{R}^{3}$," Applied Mathematics Letters, vol. 93, pp. 111-116, 2019.

[6] G. Li and H. Ye, "Existence of positive ground state solutions for the nonlinear Kirchhoff type equations in $\mathbb{R}^{3}$," Journal of 
Differential Equations, vol. 257, no. 2, article S0022039614001557, pp. 566-600, 2014.

[7] D. Lü, "A note on Kirchhoff-type equations with Hartree-type nonlinearities," Nonlinear Analysis, vol. 99, pp. 35-48, 2014.

[8] D. Naimen, "The critical problem of Kirchhoff type elliptic equations in dimension four," Journal of Differential Equations, vol. 257, no. 4, pp. 1168-1193, 2014.

[9] J. Nie, "Existence and multiplicity of nontrivial solutions for a class of Schrödinger- Kirchhoff-type equations," Journal of Mathematical Analysis and Applications, vol. 417, no. 1, pp. 65-79, 2014.

[10] X. Qian and J. Chen, "Existence of multiple solutions and estimates of extremal values for a Kirchhoff type problem with fast increasing weight and critical nonlinearity," Electronic Journal of Differential Equations, vol. 144, pp. 1-19, 2018.

[11] X. Qian and W. Chao, "Positive solutions for a Kirchhoff type problem with fast increasing weight and critical nonlinearity," Electronic Journal of Qualitative Theory of Differential Equations, vol. 27, pp. 1-17, 2019.

[12] Y. Sun and X. Liu, "Existence of positive solutions for Kirchhoff type problems with critical exponent," Journal of Partial Differential Equations, vol. 25, pp. 187-198, 2012.

[13] X. Wu, "Existence of nontrivial solutions and high energy solutions for Schrödinger-Kirchhoff-type equations in $\mathbb{R}^{N}$," Nonlinear Analysis Real World Applications, vol. 12, no. 2, pp. 1278-1287, 2011.

[14] Q. Xie, S. Ma, and X. Zhang, "Bound state solutions of Kirchhoff type problems with critical exponent," Journal of Differential Equations, vol. 261, no. 2, pp. 890-924, 2016.

[15] G. Yin and J. Liu, "Existence and multiplicity of nontrivial solutions for a nonlocal problem," Boundary Value Problem, vol. 26, pp. 1-7, 2015.

[16] C. Lei, J. Liao, and H. Suo, "Multiple positive solutions for a class of nonlocal problems involving a sign-changing potential," Electronic Journal of Differential Equations, vol. 9, pp. 1-8, 2017.

[17] C. Lei, C. Chu, and H. Suo, "Positive solutions for a nonlocal problem with singularity," Electronic Journal of Differential Equations, vol. 85, pp. 1-9, 2017.

[18] X. Qian and W. Chao, "Existence of positive solutions for nonlocal problems with indefinite nonlinearity," Boundary Value Problem, vol. 40, pp. 1-13, 2020.

[19] X. Qian and W. Chao, "Existence and concentration of ground state solutions for a class of nonlocal problem in $\mathbb{R}^{N}$," Nonlinear Analysis, vol. 203, no. 112170, pp. 1-15, 2021.

[20] Y. Wang, H. Suo, and C. Lei, "Multiple positive solutions for a nonlocal problem involving critical exponent," Electronic Journal of Differential Equations, vol. 275, pp. 1-11, 2017.

[21] Y. Wang and X. Yang, "Infinitely many solutions for a new Kirchhoff-type equation with subcritical exponent," Applicable Analysis, vol. 4, pp. 1-14, 2020.

[22] J. Zhang and Z. Zhang, "Existence of nontrivial solution for a nonlocal problem with subcritical nonlinearity," Advances in Difference Equations, vol. 359, 8 pages, 2018.
[23] W. Lu, The Variational Method in Differential Equation, Sichuan University Press, Sichun, 1995.

[24] A. Ambrosetti and P. H. Rabinowitz, "Dual variational methods in critical point theory and applications," Journal of Functional Analysis, vol. 14, no. 4, pp. 349-381, 1973.

[25] P. H. Rabinowitz, Minimax Methods in Critical Points Theory with Applications to Differential Equation, American Mathematical Society, 1986. 